\title{
Infants' Sleep Onset Crying: Learned Behavior or Evolutionary Adaptation
}

Eldar T.Hasanov

\begin{abstract}
Why do infants persistently cry when they drift off to sleep? Is sleep onset crying a learned behavior or evolutionary adaptation? Are there any evolutionary advantages behind this behavior? At some point in evolutionary history, the ancestors of humans left the trees and started terrestrial way of life. Along with many benefits that allowed hominins to eventually become modern humans, this transition made early humans more vulnerable to predation and other dangers associated with the tree-to-ground transition. The risks were particularly high for infants and young children, because unlike many other animals they are not self-reliant for a long time after their birth. Driven by evolutionary pressure, infants' sleep onset crying evolved to raise their survival probability by ensuring that their caregivers are in close proximity when they are asleep. This trait became heritable and prevalent as a result of natural selection for its capacity to repeatedly prevent threatening events after the tree-to-ground transition. Alternative behaviorist explanations argue that behavioral insomnia in childhood is a learned behavior that should be extinguished by various "cry-it-out" techniques. However, if this behavior is not acquired but genetically programmed, then according to attachment theory the attempts to obstruct it may have long-term negative effects on mechanisms of social bonding.
\end{abstract}


The inability or refusal to fall asleep in the absence of caregivers is a common behavioral issue during early childhood. It is characterized by crying just before falling asleep and represents one of the most difficult problems parents face. It involves difficulties initiating and maintaining sleep without caregivers' proximity to the child, frequently resulting in inadequate sleep and often leading to an array of negative effects for both the child and the child's family.

So why is it that just as infants start to drift off to sleep they suddenly start to cry? What are the evolutionary advantages of this behavior?

Primates are vulnerable when sleeping because their ability to notice predators is decreased. For many primates, sleeping in tree nests is a way to stay safe from predators during nighttime and the security of sleeping sites promoted increased sleep intensity, which in turn led to an increase in cognitive abilities. ${ }^{1}$ This was a major leap forward in hominin evolution.

When early hominins left the trees to start walking long distances across the land, they transitioned from nest-building in trees to sleeping on the ground. This second major step helped to enhance the quality and quantity of hominin sleep, which in turn may have promoted waking survival, supported creativity and innovation, and aided the consolidation of procedural memories. $^{2}$

Other benefits of this transition included more efficient walking for long distances using upright posture and freeing hands for using tools and carrying food, reducing skin exposure to the tropical sun by helping regulate body temperature, ${ }^{3}$ improving the visual range by elevating eyeposition and adapting to life on the savanna and other ecosystems. ${ }^{4}$

At the same time, human ancestors had to face new risks and challenges associated with a more terrestrial way of life. The risks were particularly high for infants and young children, since in 
those environments predation on the young was prevalent. Unlike many other animals, human infants are for the most part defenseless. Many savanna animals can start walking on their own within hours of birth, whereas human infants are not able to do so until about one year and are in further danger because they are unable to cling to their mothers' fur. Early human infants were especially vulnerable to predation when ground sleeping, which could come in all shapes and sizes. Along with predators currently living in the African savanna, ranging from big carnivores to venomous scorpions and spiders, there were even more deadly animals in the Paleolithic Period that included giant hyenas (Pachycrocuta), giant bears (Agriotherium), crocodiles (Crocodylus Anthropophagus) and other animals that later became extinct. In addition, there were hostile elements among their own and related species.

In order to survive in this environment, it was vital for infants and young children to be near their caretakers. As a consequence, infants' persistent crying during the moments of drifting off to sleep was evolutionarily designed to alert caregivers about their location before they fall asleep. Otherwise, after falling asleep the decreased ability to react to sounds would make it difficult to find them, especially during the darkness of night before controlled use of fire for artificial light was discovered. Without the use of fire it was difficult to ward off predatory animals at night, use dark caves for shelter, or to communicate with members of their own group. In these circumstances, infant crying associated with sleep onset and night-wakings were designed to help caregivers maintain close proximity with their infants and young children. It was an evolutionary adaptation in early human society when hominins had no settled home and constantly moved from place to place as a way of obtaining food in rugged, complex, and hostile environments. During tree-to-ground transition, this adaptation increased the survival probability of infants who spend most of their time ground sleeping. It was an evolutionary 
defense mechanism shaped by natural selection for its capacity to repeatedly prevent threatening events and helped hominins adapt to a new environment.

This adaptation also had survival value for children after acquiring locomotion skills, such as crawling and walking. Children could explore their surroundings, however, when feeling tired and drowsy their crying would alert the caregivers to their current location. This separation cry that enables a mother to locate her lost offspring might have been one of the first examples of mammalian vocalization. ${ }^{5}$

From the point of view of modern parents, their infants are totally safe sleeping alone in their rooms, so they may consider infants' persistent proximity-seeking crying and inability to fall asleep by themselves as annoying and irrational. But from the evolutionarily-devised infants' point of view, they are left alone unprotected and vulnerable in the middle of the night in a wilderness full of predators and other dangers. Even though the hostile environment that infants adapted to since has changed, the infants themselves may still carry the same genes responsible for this behavior. Human infant bedtime crying evolved as a ontogenetic adaptation that helps infants survive in their current life stage and is gradually discarded when they become older, similar to the process of imprinting in some animals. ${ }^{6}$

The dreams of modern children may present possible clues about the origins of evolutionary adaptations of early hominins. Analysis of children's dream reports shows that animals are the most common subject of children's dreams, although the wild and dangerous animals in their dreams tend to be those that are not often encountered in children's actual lives, such as monsters, bears, wolves, snakes, gorillas, tigers, lions, and biting insects. On the other hand, college students and adults have a much lower percentage of animal dreams, instead dreaming 
more of pet and farm animals encountered in real life, ${ }^{7}$ and children's dreams also had higher rates of aggression involving animals. These findings can be interpreted in terms of a threat simulation theory, which states that the present dream production system simulates threatening events in the ancestral environment. ${ }^{8}$ Even children who grow up in industrialized, urban environments free of nonhuman predators easily acquire a fear of dangerous animals because it may be genetically hard-wired. One study asked suburban American children about their fears and found that they do not "fear the things they have been taught to be careful about," such as "street traffic," but "claim that the things to be afraid of are mammals and reptiles (most frequently): snakes, lions, and tigers." 9

Many psychological theories affirm that experiences in infancy and early childhood shape the entire subsequent course of a human life. In particular, attachment theory states that in infants, behavior related to attachment is mainly a process of proximity seeking to a recognized attachment figure in situations of perceived danger and fear. Secure attachment is related to responsive parenting, including comforting babies when they wake up and cry at night. ${ }^{10}$

Alternative behaviorist explanations argue that behavioral insomnia in childhood is a learned inability to fall asleep. Where evolutionary psychology explains certain behaviors as evolutionary adaptations, behaviorism considers these behaviors as conditioned responses. Based on these ideas, parents are given conflicting recommendations about how to deal with sleep onset problems in early childhood. Proponents of the behaviorist point of view promote cry-it-out techniques, suggesting that parents should teach babies to soothe themselves to sleep by not responding to their cries for attention, arguing that picking them up and holding them whenever they cry only teaches them to cry more. 
However, if sleep onset crying is an innate and genetically programmed product of natural selection rather than a behavior acquired through learning, then utilizing these techniques may have long-term negative consequences for affected individuals. The frustrated instinct may take its revenge for being ignored by hijacking and damaging the same social bonding sphere that was being unintentionally neglected.

When a child does not receive an adequate reaction from a caregiver during crying and is unable to get attachment contact during the early stages of socialization, a cold unsympathetic emotional behavior is internalized within that individual. In a study that examined the relationship between attachment and sleep problems, mothers of securely attached infants had nighttime interactions that were generally more consistent, sensitive, and responsive than those of insecurely attached infants. ${ }^{11}$

A longitudinal study of 26 infant-mother pairs shows that consistency and promptness of maternal response is associated with decline in frequency and duration of infant crying. ${ }^{12}$ Another study shows that increased carrying reduces infant crying. ${ }^{13}$

According to Erik Erikson, when infants get dependable and consistent satisfaction of their basic needs, such as feeling safe and comforted, they learn to trust. ${ }^{14}$ Consistent with Erik Erikson's findings, attachment theory states that social experiences in early childhood serve as the source of attachment in childhood and in later life. ${ }^{15}$ An attachment system triggered by danger and separation activate attachment contact-seeking behavior in children, which in turn leads to caregiving behavior by adults. Recurring cycles of attachment signals, and consequent caregiving behavior, are internalized and later become the source of an inner feeling of security and social adaptability during the later stages of life. ${ }^{16}$ 
Utilizing attachment theory, and Erikson's theory of personality frameworks that explores relationships between rearing experiences formed during early critical developmental periods and later psychological development, can better help to understand the long-term impact on the infant when it finds itself unable to achieve an attachment contact during distress vocalizations and begging calls. The internalization of these negative attachment experiences may contribute to the emergence and development of antisocial behavioral traits during adulthood. ${ }^{17}$

Depending on the timing and nature of these negative experiences, in individuals with a genetic predisposition to extraversion and openness to experience these traits may tend to be manifested in unemotional callousness, lack of guilt, limited empathy, excessive need for attention or admiration, interpersonal manipulation, and indifference to social norms.

Whereas in individuals with genetic susceptibility to introversion and lower levels of openness to experience, there may be increased likelihood of developing traits such as difficulties with social communication and interaction, repetitive patterns of behavior and impaired nonverbal behaviors, social anxiety, and social isolation.

The way a child develops social inadequacy and his subsequent insufficiency and incompetence in interpersonal relationships may depend on a combination of behavioral predisposition and early experiences that have a capacity to trigger lack of empathy and communication deficits, and repressing attachment seeking sleep onset crying may be one of these triggers. 
${ }^{1}$ Samson, D. R., \& Nunn, C. L. (2015). Sleep intensity and the evolution of human cognition. Evolutionary Anthropology: Issues, News, and Reviews, 24, 225-237.

${ }^{2}$ Coolidge, F., \& Wynn, T. (2006). The effects of the tree-to-ground sleep transition in the evolution of cognition in early Homo. Before Farming, 4, 1-18

${ }^{3}$ Wheeler, P. E., "The Evolution of Bipedality and Loss of Functional Body Hair in Hominoids." Journal of Human Evolution, 13, 91-98, (1984).

${ }^{4}$ Niemitz, Carsten (2010). "The evolution of the upright posture and gait - a review and a new synthesis". Naturwissenschaften. 97 (3): 241-263. Bibcode:2010NW.....97..241N. doi:10.1007/s00114-009-06373. PMC 2819487. PMID 20127307.

${ }^{5}$ MacLean PD. In: Squire L, editor. The history of neuroscience in autobiography, vol. 2. New York: Academic Press; 1996. p. $245-75$

${ }^{6}$ Blasi, Carlos Hernandez; Bjorklund, David F. (2003). "Evolutionary Developmental Psychology: A New Tool for Better Understanding Human Ontogeny". Human Development. 46 (5): 259-281. doi:10.1159/000071935.

Retrieved March 31, 2016

${ }^{7}$ Domhoff, GW 1996. Finding meaning in dreams: A quantitative approach. New York: Plenum Press

${ }^{8}$ Revonsuo, A 2000. The reinterpretation of dreams: An evolutionary hypothesis of the function of dreaming. Behavioral and Brain Sciences 23:877-901

${ }^{9}$ Maurer, A. What children fear. The Journal of Genetic Psychology 106, 265-277 (1965)

${ }^{10}$ Cassidy J (1999). "The Nature of a Childs Ties". In Cassidy J, Shaver PR (eds.). Handbook of Attachment:Theory, Research and Clinical Applications. New York: Guilford Press. pp. 3-20. ISBN 978-1-57230$087-3$

${ }^{11}$ Elizabeth Higley, Mary Dozier. Nighttime maternal responsiveness and infant attachment on year June 2009 Attachment \& Human Development 11(4):347-63 DOI: 10.1080/14616730903016979

${ }^{12}$ Silvia M. Bell and Mary D. Salter Ainsworth Child Development Vol. 43, No. 4 (Dec., 1972), pp. 1171-1190

${ }^{13}$ Increased Carrying Reduces Infant Crying: A Randomized Controlled Trial UA Hunziker, RG Barr - Pediatrics, 1986 - Am Acad Pediatrics

${ }^{14}$ Bee, Helen; Boyd, Denise (March 2009). The Developing Child (12th ed.). Boston, MA: Pearson. ISBN 978-0205-68593-6.

${ }^{15}$ Mercer, J (2006). Understanding attachment: Parenting, child care, and emotional development. Westport, CT: Praeger Publishers. ISBN 978-0-275-98217-1. LCCN 2005019272. OCLC 61115448

${ }^{16}$ Bowlby J (1969). Attachment. Attachment and loss. Vol. I. London: Hogarth. ISBN 978-0-465-00543-7

${ }^{17}$ Perry, Bruce; Szalavitz, Maia (2017) [2006]. The Boy Who Was Raised as a Dog. New York: Basic Books. p. 123. ISBN 978-0-465-09445-5. 